\title{
Spontaneous categorizers retain more than spontaneous alphabetizers
}

\author{
SUSAN P. LUEK \\ Millersville State College, Millersville, Pennyslvania 17551 \\ and \\ GEORGE A. CICALA and JOHN P. MCLAUGHLIN \\ University of Delaware, Newark, Delaware 19711
}

\begin{abstract}
Immediate and delayed free recall of unstructured verbal materials were assessed for alphabetizers and categorizers after varying study times. Alphabetizers and categorizers did not differ in immediate recall, but retention following a 6-day interval was consistently higher for categorizers than for alphabetizers. Both immediate and delayed recall increased with study time, which did not interact with learning strategy. In the next experiment alphabetizers and categorizers learned two lists, the second of which was categorically structured or unstructured. No retroactive interference on first-list recall was produced by the learning of the structured list, but the learning of the unstructured list produced retroactive interference for the alphabetizers only. It was suggested that, although both alphabetizers and categorizers probably use their respective strategies for other verbal tasks, the categoric strategy should create less interference because specific categories are rarely encountered in successive tasks.
\end{abstract}

It is well known that retrieval from memory is dependent upon the manner in which the material was learned (Cofer, Bruce, \& Reicher, 1966; Puff, 1966; Wood, 1969). Instructions that constrain subjects' strategies usually produce poorer recall than do free recall instructions. Rather than examine the efficacy of various instruction sets, this paper explores differences that appear to exist among subjects' spontaneously chosen learning strategies. Learning strategy is defined herein as the overt ordering or organization which a subject imposes on a set of verbal stimuli. The experiments assess some performance differences associated with strategy differences and provide some understanding of the source of these performance differences.

Two such strategies were described in Mandler's (1967) work on the organization of verbal material. He reported extensively on the recall of those subjects who categorized during learning but only mentioned that a substantial number of his original subjects alphabetized the words in apparent disregard of the categorizing instructions. He did not consider these subjects any further. Mandler demonstrated that variations in categorization strategy, e.g., number of categories employed, were powerful variables in tests of recall. If such differences emerge from variants of one strategy, it seems likely that equally intriguing differences will be obtained if one compares the effects of categorizing in recall with those of another different strategy, alphabetizing.

Requests for reprints should be sent to Susan P. Luek, Department of Psychology, Byerly Hall, Millersville State College, Millersville, Pennsylvania 17551.
For subjects instructed to use an alphabetic strategy, immediate recall was measured by Zavortink and Keppel (1968) and compared to the recall of subjects given typical free recall instructions. They found that free recall instructions led to slightly inferior recall as compared to instructions to alphabetize. Mandler (1967) found that free recall instructions resulted in spontaneous alphabetizing by some subjects, and Zavortink and Keppel suggested that this was true of their free recall instructed subjects also. The present experiment attempted to identify during learning the strategy used and to assess directly the effects on recall of the learning strategy employed. The effects of practice duration on recall were also assessed for subjects employing different leaming strategies. This was done to explore the possibility that learning strategy interacts with duration of practice to determine recall. Thus, it is possible that alphabetizers who bring a ready-made strategy to the learning task may have better recall after short durations of practice than categorizers who must early in practice devise their own learning strategy. For longer practice durations, however, categorizing may result in better recall than alphabetizing.

In delayed recall, two classes of predictions were identified. One prediction was that forgetting would occur for all subjects at an equivalent rate and, consequently, any differences in immediate recall would be preserved in delayed recall. However, if it is assumed that such forgetting which occurs is due to interference (cf. Zavortink \& Keppel, 1968), differential forgetting for alphabetizers and categorizers would be predicted. By definition, an alphabetizer is one who spontaneously used that strategy in our procedures. Such a person 
might have applied that strategy to many learning opportunities that occurred between immediate and delayed tests. The subjects in this experiment were students who might reasonably be expected to have engaged in learning tasks where memory was required during the retention interval. If the alphabet can be considered to be the stimulus to which items to be learned become associated, the repeated use of the alphabetic strategy would constitute an A-B, A-C retroactive interference paradigm, which should produce maximal interference of original learning and lead to poorer delayed recall. Categorizers, on the other hand, may also employ the categoric learning strategy during the retention interval, but the specific categories used during interpolated learning should vary from task to task, thus constituting an A-B, C-D learning paradigm leading to little if any interference.

\section{EXPERIMENT I}

\section{Method}

Subjects. The subjects were drawn randomly from an introductory psychology course at the University of Delaware until six were tested in each of eight experimental conditions. Thus, the data from 48 subjects are included in this experiment. The subjects participated as part of a course requirement. The average age of the subjects was 20.5 years, and approximately equal numbers of males and females were included in the sample.

Stimulus materials. The list learned by all subjects was composed of 30 medium-frequency words from the Kučera and Francis (1967) computational analysis of English. The corpus frequencies of the words ranged from 18 to 32 , and two words were randomly selected from each numerical frequency level within this range. The list words and their corresponding corpus frequency values were lemon (18), ridge (18), essay (19), ignore (19), cheek (20), notable (20), bold (21), protein (21), empire (22), strategy (22), habit (23), whisky (23), actor (24), motel (24), cancer (25), ritual (25), peaceful (26), storm (26), acquire (27), ranch (27), dawn (28), passion (28), asleep (29), guilty (29), fence (30), virtue (30), muscles (31), territory (31), courage (32), and naked (32). Each word was typed in capital letters on a separate $3 \times 5$ in. index card.

Procedure. Each subject was tested individually in the presence of two experimenters. The subject sat at one of the long sides of a rectangular table, and the experimenters sat at the two ends. One of the experimenters instructed the subject to memorize the list of words, emphasizing that the cards could be rearranged and the words learned in any manner the subject wished.

After reading the instructions, the experimenter handed the subject the deck of cards and begain timing the study interval. The subjects were not aware that they were being timed. The subjects were given either $5,10,15$, or $20 \mathrm{~min}$ of study time, after which they were interrupted and asked to write the words. They were then dismissed, and it was implied that their participation in this experiment had ended. After a delay interval of 6 days, the subjects returned to another room of the laboratory, ostensibly to participate in a different and unrelated experiment. They were instructed to write as many of the words as they could remember from the previous list. Following the delayed recall test, the subjects were interviewed concerning their organizational strategies and informed about the purpose of the experiment.

Prior to the first recall test, the experimenters independently evaluated the organizational strategy of each subject. The subject was classified as an alphabetizer if he arranged the words in alphabetical order and as a categorizer if he arranged the words into columns or piles which were clearly not alphabetically organized, but rather which appeared in the experimenters' judgment to have included semantically related items. To obtain the 48 subjects used, it was necessary to judge the strategy of 61 students. It was observed that 37 of the students were categorizers and that the remaining 24 students were alphabetizers. The data analysis was simplified by excluding the last 13 categorizers, thus equalizing the number of subjects per group.

\section{Results and Discussion}

Perfect correspondence was obtained between the two experimenters' independent judgments of the learning strategy employed by each subject. Subjects judged to be categorizers sorted the words into 5 to 11 categories, and the category size varied between two and nine words. Only the 5-min categorizer group failed to assign all of the words into categories. The mean number of categories used by the 5-, 10-, 15-, and 20-min groups was $5.50,7.67,7.33$, and 6.17 , respectively.

All groups were scored on immediate and delayed recall, and the group means are included in Table 1, which shows that immediate recall did not differ for alphabetizers and categorizers within the same study intervals. However, Table 1 also shows that the delayed recall scores of categorizers were higher than those of the alphabetizers for each of the study intervals. Immediate and delayed recall for both organizational strategies increased directly with amount of study time. A comparison of the columns labeled "0 Days" and "6 Days" in Table 1 indicates that forgetting during the delay interval occurred in all groups.

A three-way analysis of variance was performed to assess the effects of (a) alphabetical vs. categorical organization, (b) study time, and (c) retention interval, on free recall. This analysis showed that the three main effects were significant. Recall varied directly with amount of study time, $F(3,40)=60.85, p<.001$, and inversely with length of the retention interval, $F(1,40)=$ $245.79, p<.001$. Categorizers recalled more words than alphabetizers, $F(1,40)=6.39, p<.025$, although that difference was due to differences in delayed recall, supported by the significant interaction of Learning Strategy by Recall Interval, $F(1,40)=22.80, p<.001$.

Table 1

Mean Number of Words Recalled by Alphabetizers and Categorizers in the Four Study-Time Conditions as a Function of Retention Interval

\begin{tabular}{|c|c|c|c|c|}
\hline \multirow{3}{*}{$\begin{array}{l}\text { Study Time } \\
\text { Condition }\end{array}$} & \multicolumn{4}{|c|}{ Retention Interval } \\
\hline & \multicolumn{2}{|c|}{0 Days } & \multicolumn{2}{|c|}{6 Days } \\
\hline & $\mathbf{M}$ & SD & $\mathbf{M}$ & $\mathrm{SD}$ \\
\hline & \multicolumn{4}{|c|}{ Alphabetizers } \\
\hline $5 \mathrm{~min}$ & 13.33 & 1.49 & 3.83 & 1.21 \\
\hline $10 \mathrm{~min}$ & 20.67 & 4.19 & 9.83 & 4.14 \\
\hline $15 \mathrm{~min}$ & 27.83 & 2.41 & 17.00 & 5.13 \\
\hline \multirow[t]{2}{*}{$20 \mathrm{~min}$} & 29.33 & 1.49 & 17.67 & 4.42 \\
\hline & \multicolumn{4}{|c|}{ Categorizers } \\
\hline $5 \mathrm{~min}$ & 13.50 & 1.61 & 7.50 & 2.75 \\
\hline $10 \mathrm{~min}$ & 19.67 & 3.14 & 12.17 & 3.93 \\
\hline $15 \mathrm{~min}$ & 28.33 & 2.36 & 23.83 & 5.58 \\
\hline $20 \mathrm{~min}$ & 29.33 & .94 & 24.50 & 4.96 \\
\hline
\end{tabular}


This interaction underscores the finding that, while alphabetizers and categorizers did not differ in immediate recall, the categorizers were significantly superior in delayed recall. There was no evidence for a differential effect of study time on alphabetizers and categorizers, since the Organization Strategy by Study Time interaction was not significant $(\mathrm{F}<1)$. Likewise, neither of the other two interactions approached significance.

An analysis of variance on all the immediate recall scores followed by a series of Duncan multiple-range tests (Edwards, 1968) showed that alphabetizers and categorizers within the same study conditions did not differ in immediate recall. For example, examination of Table 1 shows that, in the 5-min condition, alphabetizers recalled a mean of 13.33 words and categorizers recalled a mean of 13.50 words. In the 15 -min condition, the corresponding means were 27.83 and 28.33 .

These results suggest that the learning strategy did not produce effects on immediate recall, regardless of the amount of study time. Thus, it appears that for short study intervals, alphabetizing is no more effrcient than categorizing, and the application of a well learned structure does not lead to improved recall. Further, these results suggest that subjects who categorize are capable early in training of devising their categoric structure and learning the items as well. The failure to find that categorizers have better recall than alphabetizers after longer study periods is not consistent with the data of other investigators (Earhard, 1967; Tulving, 1962b; Zavortink \& Keppel, 1968). This is not surprising, since the results of those studies were inconsistent in finding that alphabetizers were sometimes superior and sometimes inferior to nonalphabetizers. The many procedural differences make these inconsistencies difficult to interpret.

The delayed recall means of the categorizers were consistently higher than those of the alphabetizers for the same study intervals. The column in Table 1 labeled "6 Days" shows this to be the case. An analysis of variance and subsequent Duncan multiple-range comparisons were performed on all of the delayed recall scores. Differences between alphabetizers and categorizers within the 15- and 20-min study intervals were significant $(p<.05)$. The apparent differences between alphabetizers and categorizers for the 5- and 10-min intervals were not significant $(p>.05)$.

The inferiority of the alphabetic strategy for longterm retention is interpreted as indicating the greater retroactive interference experienced by these groups during the retention interval, an interpretation suggested by the retroactive interference data of Zavortink and Keppel (1968). This interpretation rests on the assumption that alphabetizers engaged in leaming activities during the retention interval which elicited an alphabetic strategy and interfered with the recall of originallylearned items. While categorizers may have also engaged in learning activities during the retention interval, th nature of the learning strategy when applied to sul stantively different materials would elicit differer categories, and less interference would be obtainec An argument might be leveled against this interpr tation if it were assumed that the immediate test cor stituted an additional training trial and this trial wi more beneficial to categorizers than to alphabetizer: thus accounting for their superior long-term retentior While it is probably true that the test is an addition: training trial (Tulving, 1967), there is no apparer. reason for believing that this additional trial should $b$ more beneficial for categorizers than for alphabetizer:

Although it was not possible to identify objectivel the concepts which resulted in the establishment $c$ categories during learning, it was considered necessar to the above interpretations to determine for all subjec1 whether the learning strategy employed during learnin was also employed during recall. This was accomplishe by subjecting the recall protocols to an organization: analysis to detect the degree of correspondence be tween recall organization and the learning strateg employed.

A percentage alphabetizing index was devised for th measurement of alphabetic organization. A word wa considered to be alphabetized (a) if it was preceded by word which normally precedes it in the alphabet, 0 (b) if it was preceded by a word beginning with th same letter. The first word listed by subjects was alway scored as being alphabetized. It was found that for a] four study time groups of alphabetizers, an overall mea: of $95.8 \%$ of the words were alphabetized in immediat recall. In delayed recall, an overall mean of $82.1 \%$ o the words were alphabetized, with a range of betwees $73.6 \%$ and $89.4 \%$ for the four groups. The difficulty o obtaining the chance-level alphabetical organization scores made it necessary to compute appropriate ran] correlations between the actual output order and th alphabetical order of each alphabetizer's immediat and delayed recall protocol. Kendall's tau was compute when the number of words recalled was 10 or fewer and Spearman's tho was computed when the number o words recalled was 11 or more (McNemar, 1969, p. 234 All of the immediate recall rank correlations were sig nificant. To assess group differences in alphabeti organization, the immediate recall rank correlation were r-to-z transformed prior to averaging (McNemar 1969 , pp. 157-158). The mean correlations were signifi cant and ranged from .96 for the 5 -min alphabetizers to .99 for the 20 -min alphabetizers. With the exception o the 5-min alphabetizer group, the mean correlation in delayed recall were also significant. These correla tions were .71 for the 5 -min group, .88 for the 10 -mir group, .97 for the 15 -min group, and .94 for the $20-\mathrm{mir}$ group. The lower correlations in the short study interval were due to the small number of words recalled. Botl the percentage alphabetizing indexes and the ranl 
:orrelation coefficients strongly suggest that the alphasetizers did alphabetize on both the immediate and lelayed recall tests.

Categorical organization for the four groups of ategorizers was assessed with a clustering index dereloped and described by Roenker, Thompson, and Brown (1971). This index, referred to as the adjusted atio of clustering (ARC), was chosen in preference to ieveral others because (a) it has a constant upper limit of jerfect clustering, set at 1.000 , as well as a chance lustering level of 0.000 ; (b) it allows the comparison of relative amounts of clustering for different groups of subjects in different experimental situations; and (c) it does not vary with factors such as different numjers of items or different numbers of categories. In the present experiment, the categories were defined by the final groupings arranged by the categorizers during the study intervals. The ARC scores were computed for all categorizers for both immediate and delayed recall. A high degree of clustering was observed in immediate recall, with the mean ARC scores ranging from .756 of a maximum 1.000 for the 5 -min group to .988 of a maximum 1.000 for the 15 -min group. The overall mean ARC for immediate recall was .881. The delayed recall means ranged from .337 for the 5 -min group to .947 for the 20 -min group, with an overall mean of .744 . Considering the fact that chance clustering is set at 0.000 , it was found that a relatively high degree of clustering was in evidence.

It seems clear that the learning strategy identified by the experimenters during the study period was consistent with the order of recall given by the subjects in immediate and delayed recall. The observed differences in level of recall after a retention interval occurred in conjunction with the differences in order of recall and presumably with the differences in original learning strategy. It remains to be demonstrated, however, that the interference interpretation of these differences is the best available interpretation and that, for example, differences in delayed recall are not due to differential practice experienced during the immediate test of recall.

\section{EXPERIMENT II}

It was suggested that the greater degree of forgetting by alphabetizers in Experiement I may have been due to retroactive interference (RI) which resulted from these subjects' use of the alphabetic strategy for other verbal tasks during the retention interval. The categorizers probably experienced considerably less RI, due to the nature of their organizational strategy. That is, it is quite possible that the categorizers employed various categoric organizations for verbal learning tasks during the retention interval; however, it is highly unlikely that the actual categories they employed in the interim were similar to those they used during the experiment. The most direct way to test these assumptions would be to obtain data on the types of learning activities that categorizers and alphabetizers engaged in during the retention interval. The difficulty of obtaining these data while also maintaining the ploy that the subjects would not be retested precluded this approach.

It was therefore decided to test the effects of consonant and dissonant organizational strategies between original learning (OL) and interpolated learning (IL) to determine whether learning with an alphabetic strategy would suffer greater interference when that strategy was employed on an IL task. This is precisely what was demonstrated in the Zavortink and Keppel (1968) experiment for subjects who were instructed to use an alphabetic strategy. It remains to be demonstrated that such RI effects obtain when subjects spontaneously adopt the alphabetic learning strategy, since it is possible, although unlikely, that less RI would be produced in subjects who naturally employ the alphabetic strategy. This experiment required the interpolated learning of structured and unstructured verbal materials for subjects whose OL indicated the use of categoric or alphabetic organization. It was predicted that unstructured IL would produce more RI for alphabetizers than for categorizers and that categorically structured materials would produce little RI for either of these groups. Of course this latter prediction rests upon the testable assumption that alphabetizers use a categorizing strategy for the categorically structured IL task. The experiment was also designed to provide instances where potential sources of external interference would be minimized and where the potential differential effects of the immediate recall test on the delayed recall of alphabetizers and categorizers would be assessed directly.

\section{Method}

Subjects. Thirty-six University of Delaware undergraduates served as subjects for this experiment. Forty-six students were tested, but 10 were excluded for reasons to be discussed below. Approximately equal numbers of males and females were included in the sample, and the average age of the subjects was 19.2 years.

Stimulus materials. The original list learned by all subjects consisted of 30 nouns from the Kučera and Francis (1967) norms. Two criteria were used in the selection of the words: (a) The corpus frequencies of the words ranged from 6 to 20 , with two words selected from each numerical frequency level within this range, and (b) each of the letters of the alphabet from " $A$ " to " $W$ " was represented at least once. The original list words and their corresponding corpus frequency values were towel (6), ribbons (6), photography (7), sword (7), diamond (8), umbrella (8), beans (9), chef (9), balloon (10), wool (10), airplane (11), preacher (11), elevator (12), therapy (12), copper (13), journalism (13), kiss (17), neighborhoods (17), lamp (18), lemon (18), ambition (19), guitar (19), hatred (20), and quarrel (20). A 30-noun interpolated list was constructed according to the same criteria used for the original list, with the added restriction that none of the original list words appeared on this interpolated list. Since the nouns were not selected on the basis of their membership in identifiable taxonomic categories, the list was considered unstructured. The unstructured interpolated list words and their corpus frequencies were economist (6), hostility (6), garbage (7), utilities $(7)$, bourbon (8), debris (8), hillside (9), locker (9), costume (10), robbery (10), interim 
(11), toys (11), atlas (12), privacy (12), moonlight (13), virus (13), fantasy (14), lightning (14), needle (15), scholar (15), miracle (16), opium (16), basket (17), quote (17), classroom (18), knight (18), figs (19), string (19), jungle (20), and wit (20). It should be clear that the median Kučera-Francis frequency of both lists was 13 . Another 30 -noun interpolated list was composed of three nouns from each of 10 taxonomic categories and was considered structured. The categories which appeared on this list were selected such that no word from the original list could be considered a member of any of the categories. The category words were randomly chosen from the eight most common instances of each category, as listed in the Battig and Montague (1969) norms. The nouns and their corresponding Kučera-Francis frequencies and category labels were hammer (9), saw (352), nails (14) (carpenters' tools); yellow (55), red (197), purple (13) (colors); trout (4), herring (2), perch (1) (fish); biology (7), astronomy (24), physics (22) (sciences); Russia (72), England (154), Italy (34) (countries); hour (144), day (686), year (660) (units of time); Duke (0), Princeton (7), Harvard (34) (universities); daisy (2), lily (1), tulip (4), (flowers); football (36), tennis (15), hockey (1) (sports); legs (67), head (424), nose (60) (body parts). While some of these words were far more frequent than the items on the other lists, the median Kucera-Francis frequency of this list was 23 , which is not very different from 13 . Each word from the original list, unstructured interpolated list, and. structured interpolated list was typed in capital letters on a separate $3 \times 5$ in. index card.

Procedure. As in Experiment $I$, the subjects were tested individually, although only one experimenter was considered necessary to identify the subjects' learning strategy as either alphabetic or categoric. The experimenter read the same instructions used in Experiment $I$, handed the subject the deck of original list cards, and began timing the 10 -min study interval. Each subject was then given a 3-min written test for immediate iccall of the original list.

At the conclusion of immediate OL recall, alphabetizers and categorizers were randomly assigned to one of the experimental groups or to the control group. Each experimental subject was given either the unstructured IL deck or the structured IL deck. Within a deck, the cards were ordered randomly. The experimenter instructed each unstructured IL subject to learn the words in any desired manner. To induce a categorizing set in the structured IL subjects, the experimenter mentioned that the list contained categories. Experimental subjects were allotted $10 \mathrm{~min}$ for IL followed by $3 \mathrm{~min}$ of written IL recall. During IL the experimenter noted the learning strategies used by each subject. The control (no-IL) subjects spent a total of $13 \mathrm{~min}$ working arithmetic problems to prevent their rehearsal of the original list. The arithmetic test used was the Differential Aptitude Test of Numerical Ability-Form A (Bennett, Seashore, \& Wesman, 1947). Following this 13-min interval, a delayed recall test for OL was administered.

A total of six conditions was studied, four experimental and two control. The conditions were defined by the combination of the variety of interpolated activity and the subjective learning strategies used by the subjects. Thus, the six groups were unstructured IL alphabetizers, unstructured IL categorizers, structured IL alphabetizers, structured IL categorizers, no-IL alphabetizers, and no-IL categorizers.

Testing of subjects continued until six were in each condition. A total of 46 students was tested to obtain the 36 subjects used. Twenty-six of the students were categorizers and 18 students were alphabetizers. The remaining two students failed to rearrange the words, so their subjective learning strategies could not be identified. Their data were excluded from further consideration. To simplify the data analysis, the group sizes were equalized by the elimination of the last eight categorizers tested.

\section{Results and Discussion}

Immediate and delayed $\mathrm{OL}$ recall scores for the six groups are shown in Table 2. All groups seemed similar in level of immediate recall, but in delayed recall twc groups, the unstructured IL alphabetizers and cate. gorizers, appeared lower than the remaining groups. Consider the immediate recall data first. The immediate recall scores of all alphabetizers were not reliably differ. ent from those of categorizers, $t(34)=1.01$. This result was, of course, consistent with the results of Experiment I.

An analysis of delayed OL recall scores confirmed the visual impression that differences existed among the six groups, $F(5,30)=5.34, p<.001$. Retroactive inter. ference effects for the structured and unstructured IL alphabetizers were assessed by comparing the delayed recall mean for each of these groups with the mean of the no-IL (control) alphabetizers with Duncan multiple. range tests. A parallel set of comparisons was made for the categorizers. Significant RI was shown only in the scores of the unstructured IL alphabetizers, which were lower than those of the no-IL alphabetizers $(p<.001)$. Not one of the other three comparisons revealed differences that exceeded acceptable levels of reliability $(\mathrm{p}>.05)$.

Before it is possible to interpret these data as suggest. ing that alphabetizers show RI because they are in a virtual $A-B, A-C$ paradigm, it is necessary to show that they used an alphabetic strategy during IL with un. structured materials but not with structured materials. To do this, ARC scores were computed for structured IL, and rank correlation coefficients between alphabetic word order and actual output order were computed for unstructured IL. The mean ARC score for alphabetizers was .981 for structured materials, and the mean rank correlation between the alphabet and the actual output order for alphabetizers with unstructured materials was .99 . It is clear that the alphabetizers continued to use an alphabetic strategy during IL of unstructured materials but changed to a categorizing strategy for the IL of structured materials. Thus, interference can

Table 2

Mean Number of Words Recalled by Alphabetizers and Categorizers in Immediate and Delayed OL Tests

\begin{tabular}{|c|c|c|c|c|}
\hline \multirow{3}{*}{$\begin{array}{l}\text { Interpolated } \\
\text { Learning } \\
\text { Condition }\end{array}$} & \multicolumn{4}{|c|}{ OL Recall Test } \\
\hline & \multicolumn{2}{|c|}{ Immediate } & \multicolumn{2}{|c|}{ Delayed } \\
\hline & $\mathbf{M}$ & $\mathrm{SD}$ & $\mathbf{M}$ & $\mathrm{SD}$ \\
\hline & \multicolumn{4}{|c|}{ Alphabetizers } \\
\hline Unstructured IL & 27.67 & 1.11 & 16.50 & 3.69 \\
\hline Structured IL & 27.83 & 2.85 & 27.17 & 3.02 \\
\hline \multirow[t]{2}{*}{ No IL (control) } & 26.67 & 4.15 & 26.83 & 4.26 \\
\hline & \multicolumn{4}{|c|}{ Categorizers } \\
\hline Unstructured IL & 26.50 & 2.22 & 22.50 & 3.86 \\
\hline Structured IL & 26.17 & 4.22 & 25.67 & 4.68 \\
\hline No IL (control) & 26.17 & 3.48 & 25.83 & 3.89 \\
\hline
\end{tabular}


account for the failure of retention of alphabetizers when they persist in using an alphabetic strategy during IL. This suggests that an alphabetic organizational strategy, while effective for immediate tests, cannot reliably support long-term retention.

It was predicted that categorizers would persist in the categoric strategy during IL, and mean ARC scores of .953 and .885 for the structured and unstructured IL conditions, respectively, indicated that they did. Since the interpolated items were not related to those presented during OL, it can be safely assumed that they were organized into different categories. It appears reasonable to consider the OL and IL tasks as analogous to an A-B, C-D paradigm, since neither categorizer group showed significant $\mathrm{RI}$. Further, the failure to find significant RI for categorizers is consistent with the results reported by Birnbaum (1974), Shuell and Keppel (1967), and Winograd (1968). They have shown, however, that a categorizing strategy is not infallible, in that interference is produced when the category structure is the same in OL and IL. It seems reasonable to conclude that the results of Experiments I and II indicate that categorizing as a learning strategy is more effective than alphabetizing, in that it is more flexible due to the variety of possible category structures.

One other interpretation of Experiement $I$ is ruled out by the data of Experiment II. The superiority of categorizers on delayed OL recall might have been due to some sort of differential practice permitted by the test of immediate OL recall. Were that so, an analysis of differences between the control groups of Experiment II should have shown a difference in delayed OL recall. It did not $(\mathrm{p}>.05)$.

Very recently Barrett, Maier, Ekstrand, and Pellegrino (1975) reported that retention, over intervals comparable to those used in Experiment I, did not differ when alphabetic and semantic learning strategies were experimenter induced. They demonstrated that when all subjects were brought to the same performance criterion, they did not differ in their degree of long-term forgetting regardless of the learning strategy they employed. However, the alphabetizers and categorizers in the present experiments were virtually identical in immediate recall, but they showed obvious differences in delayed recall. This is not surprising if one accepts the assumption that a naturally occurring strategy has a greater likelihood of being used in the retention interval than one which is imposed by an experimenter, thus giving the opportunity for RI to occur. It is also apparent that experimenter-induced strategies can lead to differential forgetting, as illustrated by the RI effects reported by Zavortink and Keppel (1968).

While this research is primarily concerned with differences between learning strategies, the data are also important for more general issues in learning and memory. Organization is a theoretical construct typically defined by either (a) trial-to-trial consistency of output order (e.g., Tulving, 1962a) or (b) an identifiable re- lationship between output order and some temporal or content feature of the input (e.g., Tulving \& Pearlstone, 1966), or (c) some feature of input behavior, such as separation and sorting of the to-be-remembered items (Mandler, 1967). The inferred organization is thought to be composed of segregated units, e.g., subjective units (Tulving, 1962a). An enrichment of the theoretical features of such units has been pursued for some time (cf. Postman, 1972). The clear implication of the present data and those of Zavortink and Keppel (1968) is that an organization, or its constituent units, plays a stimulus role in acquisition and retention. In this sense, and in these experiments, an organization may be considered analogous to a series of functional stimuli in a more traditional paired-associates task.

\section{REFERENCES}

Barrett, T. R., Maier, W., Ekstrand, B. R., \& Pellegrino, J. W. Effects of experimenter-imposed organization on long-term forgetting. Journal of Experimental Psychology: Human Learning and Memory, 1975, 1, $480-490$.

Battig, W. F., \& Montague, W. E. Category norms for verbal items in 56 categories: $A$ replication and extension of the Connecticut category norms. Journal of Experimental Psychology Monograph, 1969, 80 (3, Pt. 2).

Bennett, G. K., Seashore, H. G., \& Wesman, A. G. Differential aptitude test of numerical ability (Form A). New York: The Psychological Corporation, 1947.

Birnanum, I. M. Category similarity and retroactive inhibition in free recall. Journal of Experimental Psychology, 1974, 102, 1147-1149.

Cofer, C. N., Bruce, D. R., \& Reicher, G. M. Clustering in free recall as a function of certain methodological variations. Journal of Experimental Psychology, 1966, 71, 858-866.

EARHARD, M. The facilitation of memorization by alphabetic instructions. Canadian Journal of Psychology, 1967, 21. 15-24.

EDWARDs. A. L. Experimental design in psychological research. New York: Holt, Rinehart, \& Winston, 1968.

Kučera, H., \& Francis, W. N. Computational analysis of present-day American English. Providence, R. I: Brown University Press, 1967.

MANDLER G. Organization and memory. In K. W. Spence \& J. T. Spence (Eds.). The psychology of learning and motivation (Vol. 1). New York: Academic Press, 1967.

MCNemar, Q. Psychological statistics. New York: Wiley, 1969.

Postman, L. A pragmatic view of organization theory. In E. Tulving \& W. Donaldson (Eds.), Organization of memory. New York: Academic Press, 1972.

PufF, C. R. Clustering as a function of the sequential organization of stimulus word lists. Joumal of Verbal Leaming and Verbal Behavior, 1966, 5, 503-506.

Roenker, D. L., Thompson, C. P., \& Brown, S. C. Comparison of measures for the estimation of clustering in free recall. Psychological Bulletin, 1971, 76, 45-48.

ShUELl, T. J., \& KePpel, G. Retroactive inhibition as a function of learning method. Joumal of Experimental Psychology, 1967, 75, 457-463.

TulviNG, E. Subjective organization in free recall of "unrelated" words. Psychological Review, 1962, 69, 344-354. (a)

Tulving, E. The effect of alphabetical subjective organization memorizing unrelated words. Canadian Journal of Psychology, 1962, 16, 185-191. (b) 
Tulvi:sc. E. The effects of presentation and recall of material in free-recall learning. Journal of Verbal Learning and Verbal Behavior. 1967, 6. 175-184.

Tulving. E., \& Pearlstone, Z. Availability versus accessibility of information in memory for words. Journal of Verbal Learning and Verbal Behavior, 1966, 5, 381-391.

WINOGRAD, E. List differentiation, recall, and category similarity. Journal of Experimental Psychology, 1968, 78. 510-515.
Wood, G. Retrieval cues and the accessibility of higher-orde memory units in multitrial free recall. Journal of Verba Leaming and Verbal Behavior, 1969, 8, $782-789$.

ZAvortink, B., \& KEPPEL, G. Retroactive inhibition in free recall learning with alphabetical cues. Joumal of Experimenta Psychology, 1968, 78, 617-624.

(Received for publication August 18, 1975; revision accepted November 24,1975 .) 